\title{
Spectral clustering steered low-rank representation for subspace segmentation
}

\author{
Lai Wei ${ }^{\mathrm{a}, *}$, Xiaofeng Wang ${ }^{\mathrm{a}}$, Jun Yin ${ }^{\mathrm{a}}$, Aihua $\mathrm{Wu}^{\mathrm{a}}$ \\ ${ }^{a}$ Department of Computer Science, Shanghai Maritime University, Haigang Avenue \\ 1550, Shanghai, China.
}

\begin{abstract}
Low-rank representation (LRR)and its variations have achieved great successes in subspace segmentation tasks. However, the segmentation processes of the existing LRR-related methods are all divided into two separated steps: affinity graphs construction and segmentation results obtainment. In the second step, normalize cut (Ncut) algorithm is used to get the final results based on the constructed graphs. This implies that the affinity graphs obtained by LRR-related algorithms may not be most suitable for Ncut, and the best results are not guaranteed to be achieved. In this paper, we propose a spectral clustering steered LRR representation algorithm (SCSLRR) which combines the objection functions of Ncut, K-means and LRR together. By solving a joint optimization problem, SCSLRR is able to find low-rank affinity matrices which are most beneficial for Ncut to get best segmentation results. The extensive experiments of subspace segmentation on several benchmark datasets show that SCSLRR dominates the related methods.
\end{abstract}

Keywords:

subspace segmentation, sparse representation, low-rank representation,

\footnotetext{
${ }^{*}$ Corresponding author. Tel. 86-21-38282826, fax. 86-21-38282800

Email addresses: weilai@shmtu.edu.cn (Lai Wei), xfwang@shmtu.edu.cn (Xiaofeng Wang), junyin@shmtu.edu.cn (Jun Yin), ahwu@shmtu.edu.cn (Aihua Wu)

Preprint submitted to JVCI

March 14, 2016
}

(C) 2016. This manuscript version is made available under the Elsevier user license http://www.elsevier.com/open-access/userlicense/1.0/ 
normalize cut

\section{Introduction}

In computer vision and image processing fields, high-dimensional data is usually assumed to lie in a union of linear subspaces[1, 2, 3]. The goal of subspace segmentation is to partition the data samples into the corresponding subspaces where they are generated from. Over the past decades, many subspace segmentation methods have been developed. According to the comprehensive study in [4], existing works can be roughly categorized into four main groups: iterative methods $[5,6]$, algebraic methods[7, 8], statistical methods $[9,10]$ and spectral clustering based methods $[3,11,12,13$, 14, 15, 16, 21]. Among them, spectral clustering based methods have aroused a lot of interests because of their great successes achieved in many real-world applications such as motion segmentation, image restoration, and so on.

The subspace segmentation process of a spectral clustering based method can be decomposed into two steps: firstly, for a group of data samples, an affinity graph is constructed; secondly, a spectral clustering algorithm such as normalized cut (Ncut)[17] is used to obtained the segmentation solution based on the constructed graph. It has been known that the performances of spectral clustering based methods heavily depend on whether or not the constructed affinity graphs can reveal the intrinsic structures of data sets. Over the past decades, K-nearest-neighbor (KNN)[18] may be the most frequently used graph-construction method. But it usually fails to discover the structures of complex data sets[3, 11, 19]. Because of the excellent properties such as robust to noisy data and so on, coding methods[20, 45, 46, 47, 48, 49] are recently adopted for graph construction. Among them, sparse representation (SR)[20] and its extensions[48, 49] may be the most popular ones. For 
instance, by using SR, Elhamifar et al. proposed a novel subspace segmentation algorithm, termed sparse space clustering (SSC)[21]. As reported in $[11,21]$, SSC dominates the related works in motion segmentation experiments. However, Liu et al. pointed out that the $l_{1}$-norm graphs used in SSC were insufficient for discovering the global structures of data sets[3], so that the performance of SSC would degenerate sharply when data was grossly corrupted. For the sake of remedying the limitations in SSC, Liu et al. devised a low-rank representation (LRR) algorithm[3, 14]. LRR finds the representation coefficient vector for each data sample jointly, and forces representation coefficient matrix to have minimal nuclear norm. The minimal nuclear norm constraint makes the coefficient matrix be able to reveal the global structure for a corrupted data set. Consequently, LRR shows more better performances than those of SSC in subspace segmentation tasks.

Inspired by LRR, many low-rank based subspace segmentation algorithms have been proposed. Through comparative studies, we can divide the existing low-rank related works into three categories:

1. Add more constraints on coefficient matrices. Zhuang et al. introduced sparse and non-negative constraints into LRR and presented a nonnegative low-rank and sparse representation (NNLRSR)[22]. Zhao et al. used $l_{2,1}$ constraints to make coefficient matrices be group sparse[23]. Li et al. added a b-matching constraint term into the objective function of LRR, so that the constructed affinity graphs would be symmetric[24]. Moreover, Tang et al. designed a structure-constrained low-rank representation (SCLRR) and claimed that NNLRSR was actually a special case of SCLRR[25]. Lu et al. considered that nearby samples should have similar coefficient vectors, hence they introduced a Laplacian regularized into LRR and presented a graphregularized LRR model, termed GLRR[26]. Yin et al. combined GLRR 
and NNLRSR together and put forward a Laplacian-regularized NNLRSR (called NSLLRR)[43]. These methods are shown to be more powerful to discover intrinsic structures for different kinds of data sets than LRR.

2. Improve the robustness of LRR. One one hand, LRR needs sufficient data to ensure good performance. However, the numbers of data points in real applications are usually small. Liu et al. proposed a kind of latent low-rank representation (LatLRR) algorithm which can use hidden data to improve the performance of LRR[27]. Similar to NSLLRR, Yin et al. added a Laplacian regularizer into the objective function of LatLRR and proposed a dual graph regularized latent low-rank representation[28]. Furthermore, Liu et al. presented a fixed-rank representation method (FRR) which aimed to use two matrix factors to approximate a low-rank coefficient matrix[29]. As reported in [29], FRR was more efficient and robust than LRR. On the other hand, some researchers try to decrease the sensitivity of LRR to noise data. By using a similar strategy adopted in robust sparse coding method (RSC) [30], Chen et al. proposed a robust LRR algorithm (RLRR)[31]. Vidal et al. suggested to use robust principal component analysis (RPCA)[32] to remove noise for LRR[15]. Furthermore, Jiang et al. combined RPCA and RLRR together and developed a more robust version of LRR[33].

3. Use different constraints instead of low-rank constraint. Lu et al. tried to minimize the Frobenius norms of coefficient matrices and presented a least square regression (LSR) [34]. Hu et al. proposed a kind of smooth representation clustering (SMR)[35] algorithm which hoped coefficient matrices to maintain the locality structures of original data sets. As shown in the corresponding references, these methods were much efficient than LRR and could also achieve satisfactory experimental results for subspace segmentation.

Though a lot of progresses have been made in LRR-related methods, 
there still exist a main drawback in all the above algorithms. Similar to other spectral clustering based methods, LRR-related methods decompose the subspace segmentation problems in two steps: affinity graphs construction and segmentation results obtainment. And the two steps are completely separated. This may prevent LRR-related methods to obtain the best segmentation results. In this paper, we proposed an approach termed spectral clustering algorithm steered LRR (SCSLRR) which attempted to optimize the objective function of Ncut simultaneously in the process of low-rank representation coefficient matrix achievement. Moreover, it has been known that K-means[18] is used as the final step in spectral clustering algorithms to get the final clustering results. Hence, the goal of Kmeans algorithm is also considered to be satisfied in the proposed SCSLRR algorithm. Based on these efforts, compared to other LRR-related algorithms, SCSLRR can achieve much better subspace segmentation results. We also stated the relationships between SCSLRR and some related algorithms such as SCLRR[25], GLRR[26] and structured sparse subspace clustering (S3C)[50], which will be beneficial for understanding our proposed algorithm. Finally, the subspace segmentation experiments conducted on several frequently used bench mark databases (including ORL[38], the extended Yale B[39], AR[40], MNIST(http://yann.lecun.com/exdb/mnist/)) and Hopkins 155[41] databases) will show the effectiveness of SCSLRR.

The rest of this paper is organized as follows: Section 2 briefly reviews LRR algorithm. Section 3 introduces the idea of spectral clustering steered low-rank representation (SCSLRR). We further discuss the convergence of SCLRR and the relationships between SCSLRR and some related algorithms in Section 4. The extensive experiments performed to show the effectiveness of SCSLRR are presented in Section 5. Finally, Section 6 gives the conclu- 
sions.

\section{Low-rank representation (LRR)}

LRR algorithm will be briefly reviewed firstly. Consider a set of data samples $\mathbf{X}=\left[\mathbf{x}_{1}, \mathbf{x}_{2}, \cdots, \mathbf{x}_{n}\right] \in R^{D \times n}$, and each data can be represented by the linear combination of the basis in a dictionary $\mathbf{A}=\left[\mathbf{a}_{1}, \mathbf{a}_{2}, \cdots, \mathbf{a}_{m}\right] \in$ $R^{D \times m}$, namely:

$$
\mathrm{X}=\mathrm{AZ}
$$

where $\mathbf{Z}=\left[\mathbf{z}_{1}, \mathbf{z}_{2}, \cdots, \mathbf{z}_{n}\right] \in R^{m \times n}$ is the coefficient matrix with each $\mathbf{z}_{i}$ being the representation of $\mathbf{x}_{i}$. In LRR, the data matrix $\mathbf{X}$ itself is used as the dictionary. Moreover, in real applications, data points are usually corrupted with some noise and outliers. Then, the objective function of LRR can be expressed as follows:

$$
\begin{array}{cl}
\min _{\mathbf{Z}} & \|\mathbf{Z}\|_{*}+\lambda\|\mathbf{E}\|_{2,1}, \\
\text { s.t. } & \mathbf{X}=\mathbf{X Z}+\mathbf{E},
\end{array}
$$

where $\|\mathbf{Z}\|_{*}$ is the nuclear norm of $\mathbf{Z}[36]$, i.e., the sum of the singular values of the matrix. $\|\mathbf{E}\|_{2,1}=\sum_{j=1}^{n} \sqrt{\sum_{i=1}^{n}\left([\mathbf{E}]_{i, j}\right)^{2}}$ is the $l_{2,1}$-norm of noise $\mathbf{E}^{1}$, the parameter $\lambda>0$ is used to balance the effects of the two terms. Problem (2) can be solved by using augmented Lagrange multipliers (ALM) method[13, 14].

Once $\mathbf{Z}$ is obtained, an affinity graph $\mathbf{G}$ can be defined as $[\mathbf{G}]_{i j}=\left(\left|[\mathbf{Z}]_{i j}\right|+\right.$ $\left.\left|[\mathbf{Z}]_{j i}\right|\right) / 2$. Finally, Ncut is used to get clustering results.

\footnotetext{
${ }^{1}$ Other matrix norms could also be adopted. For example, $l_{1}$-norm, $\|\mathbf{E}\|_{1}=$ $\sum_{i=1}^{n} \sum_{j=1}^{n}\left|[\mathbf{E}]_{i j}\right|$. Frobenius norm, $\|\mathbf{E}\|_{F}=\sqrt{\sum_{i=1}^{n} \sum_{j=1}^{n}[\mathbf{E}]_{i j}^{2}} \cdot[\mathbf{E}]_{i j}$ represents the $(i, j)$ th element of matrix $\mathbf{E}$.
} 


\section{Spectral clustering steered low-rank representation}

As we described and analyzed in the previous sections, it can be found that the two steps (namely, affinity graphs construction and segmentation results obtainment) of subspace segmentation processes of LRR and its variations are totally separated. This may possibly prevent Ncut to get the best segmentation solutions based on the graphs constructed by LRR-related algorithms. In this section, we will present a spectral clustering steered low-rank representation (SCSLRR) algorithm to eliminate the drawback existed in the LRR-related algorithms.

\subsection{Motivation}

Suppose we have found a low-rank coefficient matrix $\mathbf{Z}$ for a data set $\mathbf{X}=\left[\mathbf{x}_{1}, \mathbf{x}_{2}, \cdots, \mathbf{x}_{n}\right] \in R^{D \times n}$, and $\mathbf{X}$ is drawn from $C$ clusters. Then an affinity graph $\mathbf{G}$ can be defined as $[\mathbf{G}]_{i j}=\left(\left|[\mathbf{Z}]_{i j}\right|+\left|[\mathbf{Z}]_{j i}\right|\right) / 2$. Denote a diagonal matrix $\mathbf{D}$ which satisfies $[\mathbf{D}]_{i i}=\sum_{j=1}^{n}[\mathbf{G}]_{i j}$. Then a Laplacian matrix $\mathbf{L}$ can be obtained, namely $\mathbf{L}=\mathbf{D}-\mathbf{G}$. Therefore, based on the following objective function of Ncut:

$$
\begin{array}{cl}
\min _{\mathbf{H}} & \operatorname{tr}\left(\mathbf{H L H}^{T}\right), \\
\text { s.t. } & \mathbf{H H}^{T}=\mathbf{I}_{C},
\end{array}
$$

the cluster membership matrix $\mathbf{H}=\left(\mathbf{h}_{1}, \mathbf{h}_{2}, \cdots, \mathbf{h}_{n}\right) \in R^{C \times n}$ can be achieved. Here $\mathbf{H}^{T}$ is the transpose of $\mathbf{H}, \mathbf{h}_{i}=(\overbrace{0, \cdots, 0}^{i-1}, 1, \overbrace{0, \cdots, 0}^{n-i})^{T}$ denotes the cluster which $\mathbf{x}_{i}$ belongs to. Moreover, according to the algorithmic procedure of Ncut, K-means is the final step, hence $\mathbf{H}$ should satisfy the following objective function of K-means[37]:

$$
\max _{\mathbf{H}} \operatorname{tr}\left(\mathbf{H} \mathbf{X}^{T} \mathbf{X} \mathbf{H}^{T}\right)
$$


Combine problem (3) and (4) together, we have

$$
\begin{array}{cc}
\min _{\mathbf{H}} & \frac{\operatorname{tr}\left(\mathbf{H L H}^{T}\right)}{\operatorname{tr}\left(\mathbf{H X}^{T} \mathbf{X H}^{T}\right)}, \\
\text { s.t. } & \mathbf{H H}^{T}=\mathbf{I}_{C} .
\end{array}
$$

On the other hand, suppose we have gotten the cluster membership matrix $\mathbf{H}$, then Eq. (5) can be transferred to the following formulation:

$$
\begin{aligned}
\frac{\operatorname{tr}\left(\mathbf{H L H}^{T}\right)}{\operatorname{tr}\left(\mathbf{H} \mathbf{X}^{T} \mathbf{X H}^{T}\right)} & =\beta \operatorname{tr}\left(\mathbf{H}(\mathbf{D}-\mathbf{G}) \mathbf{H}^{T}\right) \\
& =\beta \operatorname{tr}\left(\sum_{i} \mathbf{h}_{i}[\mathbf{D}]_{i i} \mathbf{h}_{i}^{T}-\sum_{i j} \mathbf{h}_{i}[\mathbf{G}]_{i j} \mathbf{h}_{j}^{T}\right) \\
& =\beta \sum_{i j}[\mathbf{G}]_{i j}\left\|\mathbf{h}_{i}-\mathbf{h}_{j}\right\|_{2}^{2} \\
& =\beta \frac{1}{2} \sum_{i j}\left(\left|[\mathbf{Z}]_{i j}\right|+\left|[\mathbf{Z}]_{j i}\right|\right)\left\|\mathbf{h}_{i}-\mathbf{h}_{j}\right\|_{2}^{2} \\
& =\beta \sum_{i j}\left|[\mathbf{Z}]_{i j}\right|\left\|\mathbf{h}_{i}-\mathbf{h}_{j}\right\|_{2}^{2} \\
& =\beta\|\mathbf{M} \odot \mathbf{Z}\|_{1},
\end{aligned}
$$

where $\beta=1 / \operatorname{tr}\left(\mathbf{H X}^{T} \mathbf{X} \mathbf{H}^{T}\right)$ and $\mathbf{M} \in R^{n \times n}$ with $[\mathbf{M}]_{i j}=\left\|\mathbf{h}_{i}-\mathbf{h}_{j}\right\|_{2}^{2} . \mathbf{M}$ actually carries the structure information of a data set. Hence, Eq. (6) becomes a structure-constrained constraint on $\mathbf{Z}[25]$.

Then, we add Eq. (6) (or Eq. (5)) into the objective function of LRR and consider the following problem:

$$
\begin{array}{cc}
\min _{\mathbf{Z , H}, \mathbf{E}} & \|\mathbf{Z}\|_{*}+\lambda_{1} f(\mathbf{Z}, \mathbf{H})+\lambda_{2}\|\mathbf{E}\|_{2,1} \\
\text { s.t. } & \mathbf{X}=\mathbf{X Z}+\mathbf{E} \\
& \mathbf{H H}^{T}=\mathbf{I}_{C}
\end{array}
$$

where $f(\mathbf{Z}, \mathbf{H})=\operatorname{tr}\left(\mathbf{H} \mathbf{L} \mathbf{H}^{T}\right) / \operatorname{tr}\left(\mathbf{H} \mathbf{X}^{T} \mathbf{X} \mathbf{H}^{T}\right)\left(\right.$ or $\left.\beta\|\mathbf{M} \odot \mathbf{Z}\|_{1}\right)$. Based on the analysis, we can see that the purpose of Eq. (7) is to find a low-rank coefficient matrix for a data set which can also help Ncut to get the best segmentation result. Hence, we term problem (7) as spectral clustering steered low-rank representation (SCSLRR). 


\subsection{Optimization}

Now we will show how to solve the problem (7). Based on the above analysis, we can see $\mathbf{Z}$ and $\mathbf{H}$ depend on each other. Hence, an iterative approach is used to optimize $\mathbf{Z}$ and $\mathbf{H}$ alternately.

Update $\mathbf{H}$ with fixed Z $\mathbf{Z}$. Suppose $\mathbf{Z}$ is obtained. Then Eq. (7) degenerates to Eq. (5). Hence, $\mathbf{H}$ can be easily obtained by concentrating the $C$ eigenvectors corresponding to the matrix $\left(\mathbf{X X}^{\mathbf{T}}\right)^{-\mathbf{1}} \mathbf{L}$ 's $C$ smallest eigenvalues, where $\mathbf{L}=\mathbf{D}-\mathbf{G}$ and $\mathbf{D}, \mathbf{G}$ are obtained by using $\mathbf{Z}$.

Update $\mathbf{Z}$ with fixed $\mathbf{H}$. Suppose $\mathbf{H}$ is obtained. Then $f(\mathbf{H}, \mathbf{Z})=$ $\beta\|\mathbf{M} \odot \mathbf{Z}\|_{1}$. Eq. (7) becomes the following optimization problem:

$$
\begin{array}{cc}
\min _{\mathbf{Z , E} \mathbf{E}} & \|\mathbf{Z}\|_{*}+\hat{\lambda_{1}}\|\mathbf{M} \odot \mathbf{Z}\|_{1}+\lambda_{2}\|\mathbf{E}\|_{2,1} \\
\text { s.t. } & \mathbf{X}=\mathbf{X Z}+\mathbf{E},
\end{array}
$$

where $\hat{\lambda_{1}}=\lambda_{1} \beta$. Eq.(8) can be solved by using augmented Lagrange multiplier (ALM) method[13, 14]. Firstly, it can be converted to the following equivalent problem:

$$
\begin{array}{cc}
\min _{\mathbf{Z}, \mathbf{J}, \mathbf{W}, \mathbf{E}} & \|\mathbf{J}\|_{*}+\hat{\lambda_{1}}\|\mathbf{M} \odot \mathbf{W}\|_{1}+\lambda_{2}\|\mathbf{E}\|_{2,1} \\
\text { s.t. } & \mathbf{X}=\mathbf{X Z}+\mathbf{E} \\
\mathbf{Z}=\mathbf{J} \\
\mathbf{Z}=\mathbf{W} .
\end{array}
$$

The augmented Lagrangian function of Eq. (9) can be expressed as follows:

$$
\begin{aligned}
& \min _{\mathbf{Z}, \mathbf{J}, \mathbf{W}, \mathbf{E}}\|\mathbf{J}\|_{*}+\hat{\lambda_{1}}\|\mathbf{M} \odot \mathbf{W}\|_{1}+\lambda_{2}\|\mathbf{E}\|_{2,1} \\
& +\left\langle\mathbf{Y}_{1}, \mathbf{X}-\mathbf{X Z}-\mathbf{E}\right\rangle+\left\langle\mathbf{Y}_{2}, \mathbf{Z}-\mathbf{J}\right\rangle+\left\langle\mathbf{Y}_{3}, \mathbf{Z}-\mathbf{W}\right\rangle \\
& +\mu / 2\left(\|\mathbf{X}-\mathbf{X Z}-\mathbf{E}\|_{F}^{2}+\|\mathbf{Z}-\mathbf{J}\|_{F}^{2}+\|\mathbf{Z}-\mathbf{W}\|_{F}^{2}\right),
\end{aligned}
$$

where $\mathbf{Y}_{1}, \mathbf{Y}_{2}$ and $\mathbf{Y}_{3}$ are Lagrange multipliers and $\mu>0$ is a parameters. Then $\mathbf{Z}, \mathbf{J}, \mathbf{W}, \mathbf{E}$ can be updated in sequence with fixed other variables. The 
precise updating schemes can be summarized as follows:

$$
\left\{\begin{aligned}
\mathbf{J}_{k+1} & =\arg \min _{\mathbf{J}_{k}} 1 / \mu_{k}\left\|\mathbf{J}_{k}\right\|_{*}+1 / 2\left\|\mathbf{J}_{k}-\left(\mathbf{Z}_{k}+\mathbf{Y}_{2}^{k} / \mu_{k}\right)\right\|_{F}^{2} \\
\mathbf{W}_{k+1} & =\arg \min _{\mathbf{W}_{k}} \hat{\lambda_{1}} / \mu_{k}\left\|\mathbf{M} \odot \mathbf{W}_{k}\right\|_{1}+1 / 2\left\|\mathbf{W}_{k}-\left(\mathbf{Z}_{k}+\mathbf{Y}_{3}^{k} / \mu_{k}\right)\right\|_{F}^{2}, \\
\mathbf{Z}_{k+1} & \left.=\left(\mathbf{X}^{T} \mathbf{X}+2 \mathbf{I}_{n}\right)^{-1}\left(\mathbf{X}^{T}\left(\mathbf{X}-\mathbf{E}_{k}+\mathbf{Y}_{1}^{k} / \mu_{k}\right)+\mathbf{J}_{k+1}-\mathbf{Y}_{2}^{k} / \mu_{k}+\mathbf{W}_{k+1}-\mathbf{Y}_{3}^{k} / \mu_{k}\right)\right) \\
\mathbf{E}_{k+1} & =\arg \min \lambda_{2} / \mu_{k}\left\|\mathbf{E}_{k}\right\|_{2,1}+1 / 2\left\|\mathbf{E}_{k}-\left(\mathbf{X}-\mathbf{X} \mathbf{Z}_{k+1}+\mathbf{Y}_{1}^{k} / \mu_{k}\right)\right\|_{F}^{2}, \\
\mathbf{Y}_{1}^{k+1} & =\mathbf{Y}_{1}^{k}+\mu_{k}\left(\mathbf{X}-\mathbf{X} \mathbf{Z}_{k+1}-\mathbf{E}_{k+1}\right), \\
\mathbf{Y}_{2}^{k+1} & =\mathbf{Y}_{2}^{k}+\mu_{k}\left(\mathbf{Z}_{k+1}-\mathbf{J}_{k+1}\right), \\
\mathbf{Y}_{3}^{k+1} & =\mathbf{Y}_{3}^{k}+\mu_{k}\left(\mathbf{Z}_{k+1}-\mathbf{W}_{k+1}\right), \\
\mu_{k+1} & =\min \left(\mu_{\max }, \rho \mu_{k}\right),
\end{aligned}\right.
$$

where $\mu_{\max }$ and $\rho$ are two positive parameters, $k$ indicates the index of iteration. The detailed derivations of $\mathbf{J}, \mathbf{W}, \mathbf{Z}, \mathbf{E}$ could be found in Appendix A.

\subsection{The algorithm}

We summarize the algorithmic procedure of SCSLRR in this section. Firstly, suppose $\mathbf{H}$ is obtained, the algorithmic procedure for solving Eq. (8) is presented in Algorithm 1.

Secondly, the complete spectral clustering steered low-rank representation (SCSLRR) algorithm is described in Algorithm 2.

\section{Further Discussion}

\subsection{The convergence of SCSLRR}

We first discuss the convergence of SCSLRR in this subsection. The following proposition can be proved.

Proposition 1: SCSLRR will converge to a local minimum by using

\section{Algorithm 2.}




\section{Algorithm 1 Computing $\mathbf{Z}$ by using ALM Input:}

Data set $\mathbf{X}=\left[\mathbf{x}_{1}, \mathbf{x}_{2}, \cdots, \mathbf{x}_{n}\right] \in R^{D \times n}$ with each column has unit $l_{2}$ norm, parameters $\lambda_{1}, \lambda_{2}$ and the weighted matrix $\mathbf{M}$ with $[\mathbf{M}]_{i j}=\left\|\mathbf{h}_{i}-\mathbf{h}_{j}\right\|_{2}^{2}$, the maximal number of iteration Maxiter;

\section{Output:}

The coefficient matrix $\mathbf{Z}^{*}$;

1: Initialize the variables and parameters, i.e., $\mathbf{J}_{0}=\mathbf{W}_{0}=\mathbf{Z}_{0}=0, \mathbf{Y}_{1}^{0}=$ $\mathbf{Y}_{2}^{0}=\mathbf{Y}_{3}^{0}=0, \mu_{0}=10^{-6}, \mu_{\max }=10^{10}, \rho=1.1, \varepsilon=10^{-8}, k=1$.

2: while $\left\|\mathbf{X}-\mathbf{X} \mathbf{Z}_{k}-\mathbf{E}_{k}\right\|_{\infty}>\varepsilon$ and $\left\|\mathbf{Z}_{k}-\mathbf{J}_{k}\right\|_{\infty}>\varepsilon$ and $\left\|\mathbf{Z}_{k}-\mathbf{W}_{k}\right\|_{\infty}>\varepsilon$ and $k<$ Maxiter do

3: $\quad$ Update $\mathbf{J}_{k}, \mathbf{W}_{k}, \mathbf{Z}_{k}, \mathbf{E}_{k}, \mathbf{Y}_{1}^{k}, \mathbf{Y}_{2}^{k}, \mathbf{Y}_{3}^{k}$ and $\mu_{k}$ by using Eq. (11);

4: end while

5: return the coefficient matrix $\mathbf{Z}^{*}=\mathbf{Z}_{k}$. 
$\overline{\text { Algorithm } 2 \text { Spectral clustering steered low-rank representation (SCSLRR) }}$ Input:

Data set $\mathbf{X}=\left[\mathbf{x}_{1}, \mathbf{x}_{2}, \cdots, \mathbf{x}_{n}\right] \in R^{D \times n}$, parameters $\lambda_{1}, \lambda_{2}$, the number of class $C$, the maximal number of iteration Maxiter.

\section{Output:}

The coefficient matrix $\mathbf{Z}^{*}$;

1: Initialize $\mathbf{Z}_{1}=\left(\mathbf{X}^{T} \mathbf{X}\right)^{-1} \mathbf{X}^{T} \mathbf{X}, \mathbf{Z}_{0}=0, t=1, \varepsilon=10^{-5}$.

2: while $\left\|\mathbf{Z}_{t}-\mathbf{Z}_{t-1}\right\|_{\infty}>\varepsilon$ and $t<$ Maxiter do

3: Compute $\mathbf{H}_{t} . \mathbf{H}_{t}$ is composed of the $C$ eigenvectors corresponding to $\mathbf{L}_{t}$ 's $C$ smallest eigenvalues, where $\mathbf{L}_{t}=\mathbf{D}_{t}-\mathbf{G}_{t} .\left[\mathbf{G}_{t}\right]_{i j}=1 / 2\left(\left[\mathbf{Z}_{t}\right]_{i j}+\right.$ $\left[\mathbf{Z}_{t}\right]_{j i}$ ), and $\mathbf{D}_{t}$ is diagonal matrix which satisfies $\left[\mathbf{D}_{t}\right]_{i i}=\sum_{j=1}^{n}\left[\mathbf{G}_{t}\right]_{i j}$;

4: $\quad$ Use Algorithm $\mathbf{1}$ to obtain $\mathbf{Z}_{t+1}$;

5: $\quad t=t+1$.

6: end while

7: return $\mathbf{Z}^{*}=\mathbf{Z}_{t}$.

Notice: $\mathbf{Z}_{1}=\left(\mathbf{X}^{T} \mathbf{X}\right)^{-1} \mathbf{X}^{T} \mathbf{X}$ is good initial value for $\mathbf{Z}$. It is the solution to the problem $\min _{\mathbf{Z}}\|\mathbf{X}-\mathbf{X Z}\|_{F}^{2}$. 
Proof: Firstly, Algorithm 1 is a typical ALM method which is convergent with mild conditions[13, 14].

Secondly, we denote the objective function of SCSLRR as $\mathbb{J}(\mathbf{Z}, \mathbf{H})$. Based on the definition of $\mathbb{J}(\mathbf{Z}, \mathbf{H})$, it can be seen that each term in $\mathbb{J}(\mathbf{Z}, \mathbf{H})$ is larger than zero, hence $\mathbb{J}(\mathbf{Z}, \mathbf{H}) \geq 0$. Then once $\mathbf{Z}_{t}$ is obtained, we have $\mathbf{H}_{t+1}=\arg \min _{\mathbf{H}} f\left(\mathbf{Z}_{t}, \mathbf{H}_{t}\right)$, hence $f\left(\mathbf{Z}_{t}, \mathbf{H}_{t+1}\right) \leq f\left(\mathbf{Z}_{t}, \mathbf{H}_{t}\right)$. Consequently, $\mathbb{J}\left(\mathbf{Z}_{t}, \mathbf{H}_{t+1}\right) \leq \mathbb{J}\left(\mathbf{Z}_{t}, \mathbf{H}_{t}\right)$. On the other hand, once $\mathbf{H}_{t+1}$ is obtained, we have $\mathbf{Z}_{t+1}=\arg \min \mathbb{J}\left(\mathbf{Z}_{t}, \mathbf{H}_{t+1}\right)$, hence $\mathbb{J}\left(\mathbf{Z}_{t+1}, \mathbf{H}_{t+1}\right) \leq \mathbb{J}\left(\mathbf{Z}_{t}, \mathbf{H}_{t+1}\right) \leq \mathbb{J}\left(\mathbf{Z}_{t}, \mathbf{H}_{t}\right)$. Hence, we can conclude that SCSLRR is convergent.

\subsection{Comparisons between SCSLRR and some related algorithms}

We make a comparative study of SCSLRR and some related algorithms, such as SCLRR[25], GLRR[26] and S3C[50].

\section{A. Comparisons with SCLRR}

We can find that if the cluster membership matrix $\mathbf{H}$ is computed, SCSLRR has a similar objective function to SCLRR. Then the difference between the two objective functions is the definition of the weighted sparse term $\|\mathbf{M} \odot \mathbf{Z}\|$. In SCLRR, $\mathbf{M}$ is computed by using the original data set $\mathbf{X}$, namely $[\mathbf{M}]_{i j}=\left\|\mathbf{x}_{i}-\mathbf{x}_{j}\right\|_{2}$. Because of the existence of noise in the original data set $\mathbf{X}, \mathbf{M}$ defined in SCLRR may not be able to indicate the truthful structure of $\mathbf{X}$. In SCSLRR, $\mathbf{M}$ is defined by using the cluster membership matrix $\mathbf{H},[\mathbf{M}]_{i j}=\left\|\mathbf{h}_{i}-\mathbf{h}_{j}\right\|_{2}$. And $\mathbf{H}$ is computed based on $\mathbf{Z}$. Because $\mathbf{Z}$ is able to reveal the structures of data sets to some extend, $\mathbf{M}$ carries more accurate cluster structure information of the data sets. Hence, the solution of SCSLRR will be inevitably superior to that of SCLRR.

\section{B. Comparisons with GLRR}

GLRR compels the representation coefficient vectors of nearby points 
should be similar to each other. So a Laplacian regularizer $\operatorname{tr}\left(\mathbf{Z L Z} \mathbf{Z}^{T}\right)$ with respect to the low-rank coefficient matrix $\mathbf{Z}$ is adopted, where the Laplacian matrix $\mathbf{L}$ is defined based on the original data set $\mathbf{X}$. Nevertheless, in our SCSLRR, $f(\mathbf{Z}, \mathbf{H})=\operatorname{tr}\left(\mathbf{H L H} \mathbf{H}^{T}\right) / \operatorname{tr}\left(\mathbf{H} \mathbf{X}^{T} \mathbf{X} \mathbf{H}^{T}\right)$ can be regard as a generalized Laplacian regularizer w.r.t $\mathbf{H}$ (Here, $\mathbf{L}$ is defined based on $\mathbf{Z}$ ). It is used to compute $\mathbf{H}$ and will be transferred to be a structure-constraint on $\mathbf{Z}$ when we are computing $\mathbf{Z}$. Hence, the goals of the two algorithms are much different. Moreover, based on our experiments, GLRR is very sensitive to the parameters and can not get satisfactory solutions with improper parameters. On the contrary, the performance of SCSLRR is very stable even when the parameters vary in relative large ranges.

\section{Comparision with S3C}

Recently, Li et al. proposed a kind of structured sparse subspace clustering algorithm (S3C) whose objective function can be expressed as follows:

$$
\begin{array}{cc}
\min _{\mathbf{Z}, \mathbf{H}, \mathbf{E}} & \|\mathbf{Z}\|_{1, \mathbf{H}}+\lambda\|\mathbf{E}\|_{2,1}, \\
\text { s.t. } & \mathbf{X}=\mathbf{X Z}+\mathbf{E}, \operatorname{diag}(\mathbf{Z})=\mathbf{0},
\end{array}
$$

where $\|\mathbf{Z}\|_{1, \mathbf{H}}=\|\mathbf{Z}\|_{1}+\alpha\|\mathbf{M} \odot \mathbf{Z}\|_{1}$ and $\mathbf{M}$ is defined as $[\mathbf{M}]_{i j}=\left\|\mathbf{h}_{i}-\mathbf{h}_{j}\right\|_{2}$. We can see that the idea of S3C is much similar to that of our SCSLRR. However, for one thing, the coefficient matrix computed by $\mathrm{S} 3 \mathrm{C}$ is not a low-rank matrix which will make the performance of S3C degenerate sharply when data is grossly corrupted[3]. For the other thing, in $\mathrm{S} 3 \mathrm{C}, \mathbf{H}$ is the solution to Ncut, namely Eq. (3). However, $\mathbf{H}$ is computed based on Eq. (5) in SCSLRR. And it simultaneously satisfies the goal of Ncut and Kmeans. As we mentioned, K-means is the final step of Ncut, its goal should be achieved. Therefore, $\mathbf{H}$ computed in SCSLRR can characterize the cluster membership of an original data set more precisely. Based on two reasons, SCSLRR outperforms S3C. 


\section{Experiments}

In this section, some subspace segmentation experiments will be performed to prove the effectiveness of SCSLRR. The related algorithms such as $\mathrm{SSC}[21], \mathrm{S} 3 \mathrm{C}[50], \operatorname{LRR}[3,14], \operatorname{SCLRR}[25]$ and GLRR[26] will be also evaluated for comparisons. Two types data sets including several image databases(ORL database[38], the extend Yale B database[39], AR database[40], MINIST database(http://yann. lecun.com/exdb/mnist/)) and Hopkins 155 motion segmentation data set[41] will be used.

The brief information of the five databases are summarized as follows:

ORL database contains 400 face images (without noise) of 40 persons. Each individual has 10 different images. These images were taken at different times, varying the lighting, facial expressions (open/closed eyes, smiling/not smiling) and facial details (glasses/no glasses).

The extended Yale B face database contains 38 human subjects and around 64 near frontal images under different illuminations per individual. In the database, some images are corrupted by shadow. We use images from first 10 classes of extended Yale B database to form a set of heavily corrupted data which contains 640 images in total. In our experiments, each image from the above two databases is resized to $32 \times 32$ pixels.

AR database consists of over 4000 face images of 126 individuals. For each individual, 26 pictures were taken in two sessions (separated by two weeks) and each section contains 13 images. These images include front view of faces with different expressions, illuminations and occlusions. In our experiments, we take the pictures from the first 20 persons (520 images) of AR. And each image is resized into $50 \times 40$. Some sample images from the databases are shown in Fig. 1(a), (b) and (c) respectively.

MNIST database has 10 subjects, corresponding to 10 handwritten digits, 


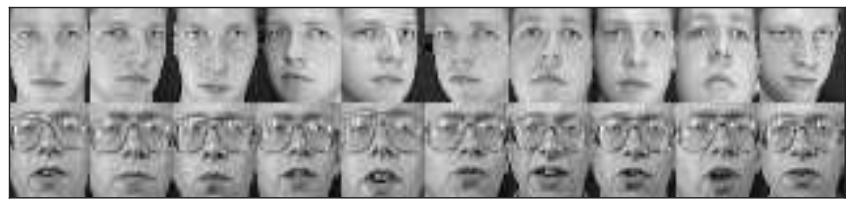

(a) Sample images from ORL database

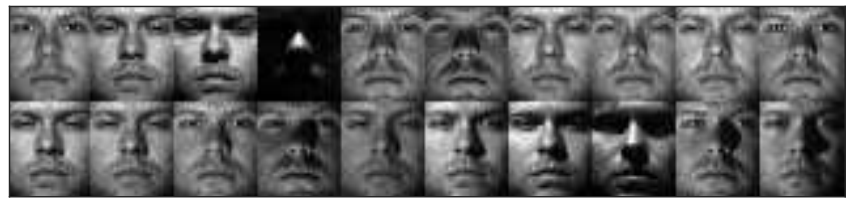

(b) Sample images from the extended Yale B database

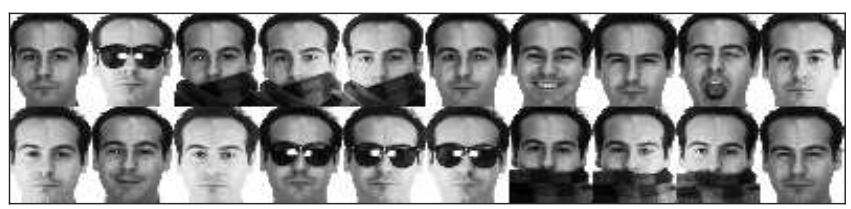

(c) Sample images from AR database

Figure 1: Sample Images from (a) ORL database, (b) the extended Yale B database, (c) AR database. 


\section{4 6565566666119798898899999 \\ (a) Sample Images of MNIST database}
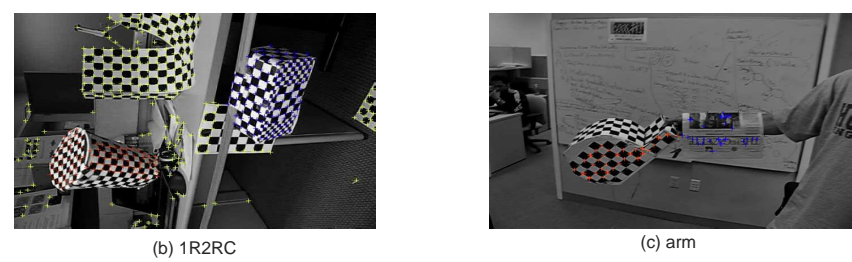

Figure 2: Sample Images of (a) MNIST database and (b),(c) Hopkins 155 motion segmentation database.

namely $0-9$. We select a subset which consists of the first 50 samples of each subject's training data set. Each image is resize to $28 \times 28$ pixels.

Hopkins155 motion segmentation database consists of 120 sequences of two motions and 35 sequences of three motions ${ }^{2}$. The features are extracted and tracked along with the motion in all frames, and errors were manually removed for each sequence. So it could be regarded that this database only contains slight corruptions. Each sequence is a sole clustering task and there are 155 clustering tasks in total. Fig. 2 presents the sample images of MNIST database and Hopkins 155 database.

Then the experiments can be divided into three perspectives: firstly, we verify the convergence of SCSLRR; secondly, we test the stability of SCSLRR with different values of parameters $\lambda_{1}, \lambda_{2}$; thirdly, we compare the

\footnotetext{
${ }^{2}$ It also contains a sequence of 5 motions which is called "dancing". We neglect this sub-database in our experiments.
} 

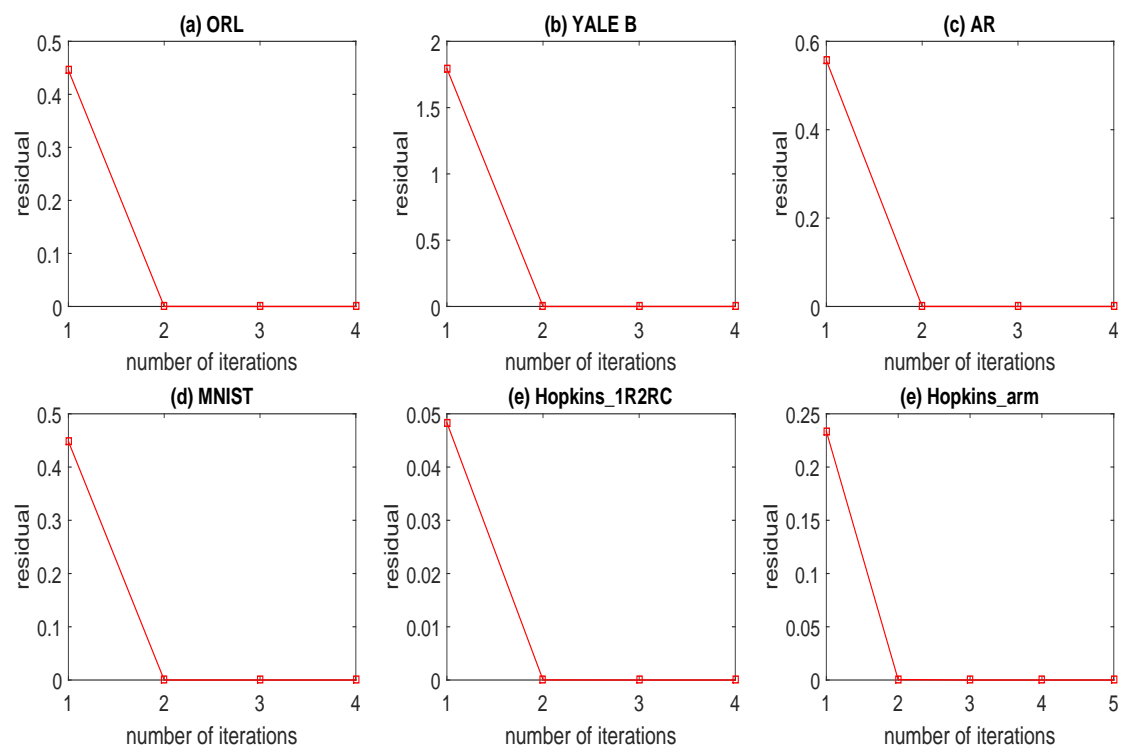

Figure 3: The convergence of SCSLRR on different databases.

performances of SCSLRR with those of related algorithms.

\subsection{Convergence of SCSLRR}

In this subsection, we try to verify the convergence of SCSLRR. For each image database, we first project the data samples into its $(n-1)$-dimensional principal subspace by using principal component analysis (PCA)[42], where $n$ is the number of data samples in corresponding database. For Hopkins 155 database, two representative sub-databases LR2RC (3 motions) and arm (2 motions) are employed. PCA is used to project the data from Hopkins 155 into 12-dimensional subspaces. We simply set the two parameters in SCSLRR $\lambda_{1}=1, \lambda_{2}=1$. Duing the experiments, the residual $e=\left\|\mathbf{Z}_{t}-\mathbf{Z}_{t-1}\right\|_{\infty}$ in each iteration is recorded. Then the experimental results are illustrated in Fig. 3 .

Obviously, from Fig. 3, we can see that the convergence rate of SCSLRR 
is very high on all the databases.

\subsection{Sensitivity to the parameters}

In this subsection, we test the sensitivity of SCSLRR to the parameters $\lambda_{1}$ and $\lambda_{2}$. We let the two parameters vary in the interval $[0.01,50]$ and record the segmentation accuracy ${ }^{3}$ obtained by SCSLRR on different databases. The databases used in the previous experiments are also adopted in these experiments. And the experimental results are illustrated in Fig.4.

Form Fig. 4, we can get several conclusions: 1) the performances of SCSLRR are much stable when $\lambda_{1}$ and $\lambda_{2}$ vary in a relative large range on Hopkins155 database; 2) when $\lambda_{1}, \lambda_{2}$ vary from 2 to 10, the performances of SCSLRR are stable on ORL and AR databases; 3) SCSLRR is also insensitivity to the variations of $\lambda_{1}, \lambda_{2}$ on the extend YALE B and MNIST databases, and achieves best results when $\lambda_{1}=1$.

\subsection{Comparisons to the related algorithms}

Now in this subsection, we will evaluate the performances of mentioned LRR-related algorithms (such as SSC[21], S3C[50], LRR[3, 14], SCLRR[25] and GLRR[26]) and show that SCSLRR is superior to the related algorithms.

\section{A. Experiments on Hopkin155 database.}

Firstly, Hopkins 155 database in used. The detailed statistics of the segmentation errors ${ }^{4}$ of the evaluated algorithms including Mean, standard deviation (Std.) and maximal error(Max.) are recorded in Table1. Based on the previous experiments, we set the two parameters in SCSLRR as $\lambda_{1}=$

\footnotetext{
${ }^{3}$ Segmentation accuracy is obtained by computing the percentage of correctly classified data vectors. In this paper, the label of a cluster is the index of the ground truth class that contributes the maximum number of samples to the cluster[14].

${ }^{4}$ segmentation error $=1$ - segmentation accuracy.
} 

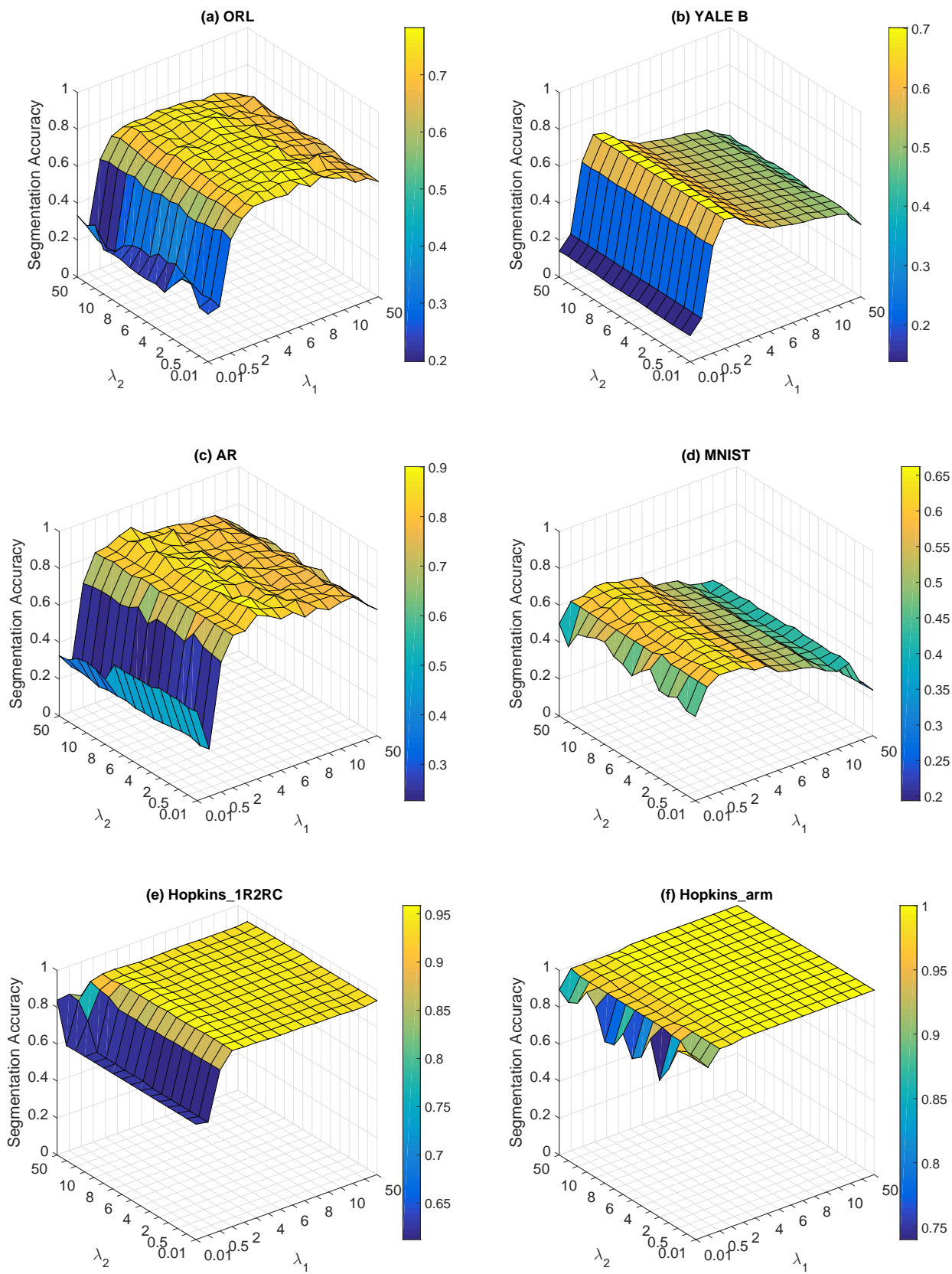

Figure 4: The sensitivity of SCSLRR to the parameters $\lambda_{1}, \lambda_{2}$ on different databases. 
Table 1: The experimental results of different algorithms on Hopkins 155 database. The optimal values of different criterion are emphasized in bold style.

\begin{tabular}{ccccccc}
\hline & SSC & S3C & LRR & SCLRR & GLRR & SCSLRR \\
Mean & 4.8 & 4.2 & 3.8 & 3.6 & 5.8 & $\mathbf{2 . 8}$ \\
Std. & 7.8 & 6.6 & 6.8 & 9.2 & 10.4 & $\mathbf{6 . 5}$ \\
Max. & 41.2 & 39.6 & 40.2 & 39.6 & 48.9 & $\mathbf{3 7 . 3}$ \\
\hline
\end{tabular}

Notice: In SSC, $\lambda=1$. In S3C, $\beta=0.01, \lambda=1$. In LRR, $\lambda=2.4$. In SCLRR, $\beta=0.01, \lambda=2$. In GLRR, $\lambda=0.0001$.

$5, \lambda_{2}=5$. And the parameters in other related algorithms are set to the best values by following the disciplines stated in the related references which are also reported in Table 1.

From Table 1, we can find that the mean, standard deviation and maximal error of segmentation error obtained by SCSLRR are all slightly better than those of other algorithms.

\section{B. Experiments on image databases}

Secondly, the four image databases are used for comparison. For each image database, we select $q$ classes data samples ( $q$ varies from 3(or 4 or 5 ) to the total number of class of data samples in each database) and project the data samples into their principal subspace (similar to the previous experiments, the dimension of principal subspace is set to $n-1, n$ is the number of samples). The six evaluated algorithms are then used to compute the corresponding segmentation accuracies. For each algorithm, we let the parameters vary in the interval $[0.001,50]$, and the values corresponding to the highest accuracy are chosen. Finally, the segmentation accuracy obtained by each algorithm versus variation of the range of number of class $q$ for each is shown in Fig. 5. 

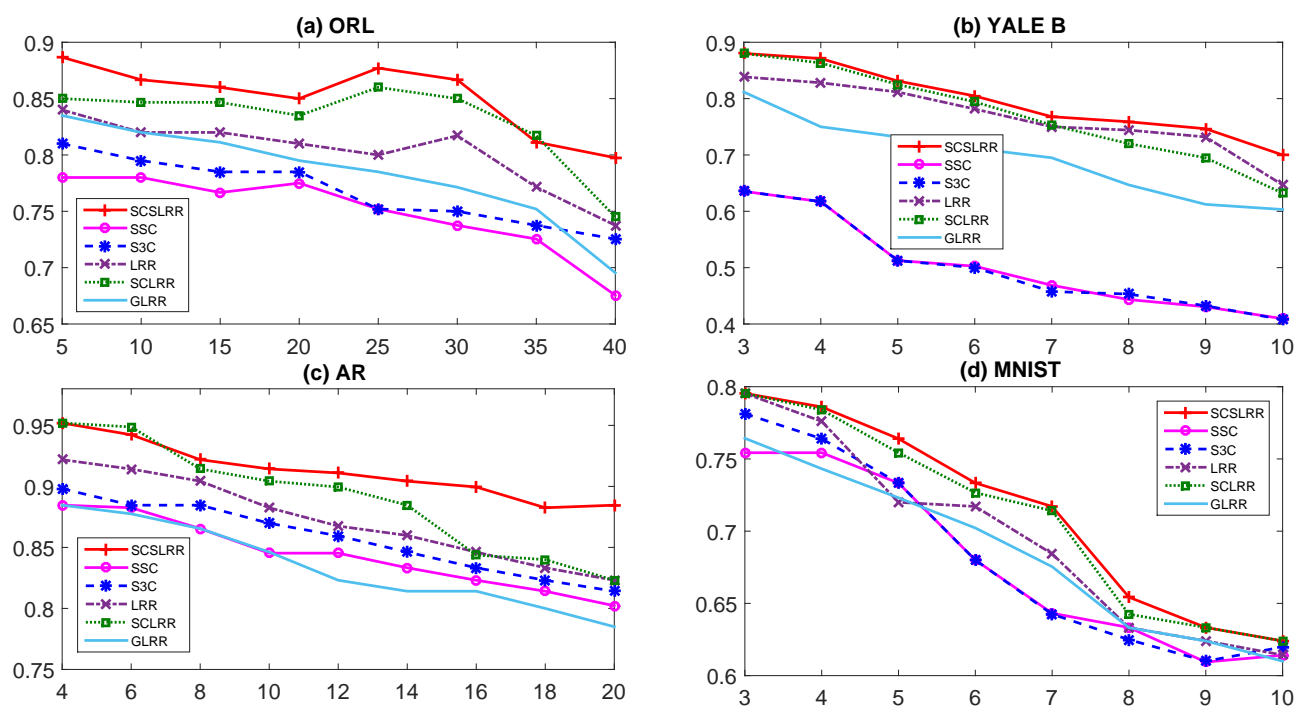

Figure 5: The segmentation accuracy obtained by each evaluated algorithm versus variation of the range of number of class on different databases, where (a) ORL, (b) the extended YALE B, (c) AR, (d) MNIST. The horizontal axis of each image represents the number of class and the vertical axis denotes the segmentation accuracy. 
From Fig. 5, we can get several conclusions: 1) SCSLRR always achieves best segmentation accuracies on the four databases when the number of class varies; 2) the results of SCLRR and S3C are better those of LRR and SSC respectively, which can prove that the locality constraint is useful for improving the performance of LRR and SSC. 3) LRR-related algorithms are superior to SSC and S3C. Especially on the extend Yale B database, LRR related algorithms obtain much better results than SSC and S3C. The reason may be some data samples in the extend Yale B database are heavily corrupted, hence the $l_{1}$ graphs used in SSC and S3C can not reveal the true intrinsic structure of the database.

Furthermore, we also test the impact of dimension of principal subspaces on the performance each evaluated algorithm. In these experiments, all the data samples in the four databases will be adopted. The dimension of the principal subspaces computed by PCA will vary in $[40,400]$ for the four image databases. And the segmentation accuracy obtained by each algorithm in the corresponding principal subspace is recorded in Fig. 6 .

From Fig. 6, we can see that 1) SCSLRR still outperforms other related algorithms in most cases on the four databases; 2) and its performance is stable when the dimension of the principal subspaces changes; 3) LRR-related algorithms are superior to SR-related algorithm.

Finally, based on all these experiments, we can conclude that SCSLRR is an efficient and effective algorithm for subspace segmentation tasks.

\section{Conclusion}

LRR and its variations have shown great abilities in handling subspace segmentation tasks. However, all the existing LRR-related methods have not considered to satisfy the objective function of Ncut algorithm during the affin- 
(a) ORL

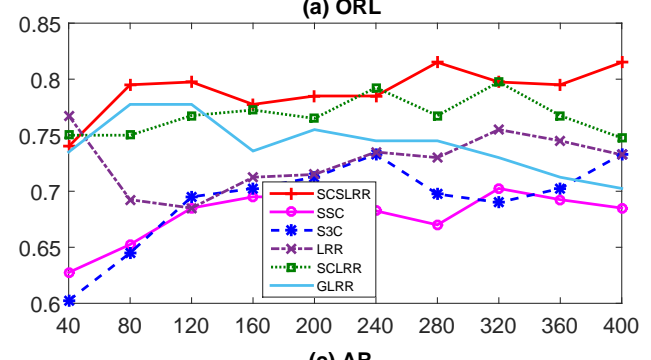

(c) AR

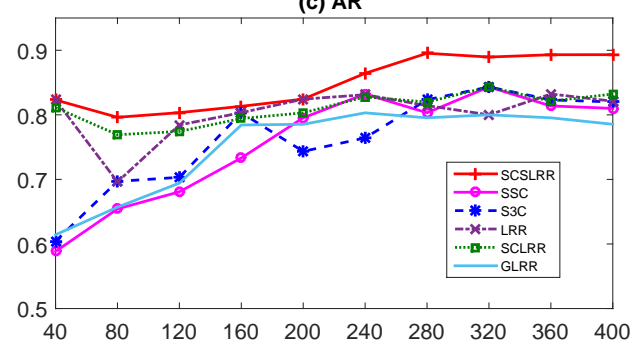

(b) YALE B

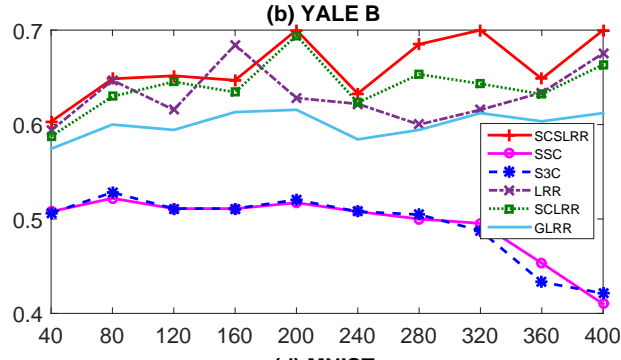

(d) MNIST

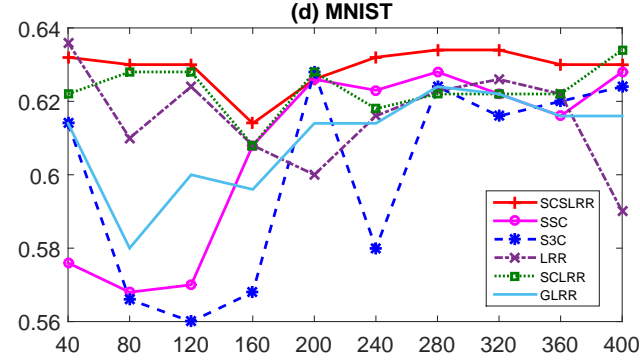

Figure 6: The segmentation accuracy obtained by each evaluated algorithm versus variation of dimension of the principal subspaces of different databases, where (a) ORL, (b) the extended YALE B, (c) AR, (d) MNIST. The horizontal axis of each image represents the dimension of the principal subspace and the vertical axis denotes the segmentation accuracy. 
ity graphs construction. Hence, the existing LRR-related methods may not be able to achieve best segmentation results. In this paper, we put forward a spectral clustering steered low-rank representation algorithms(SCSLRR) for subspace segmentation. SCSLRR combines N-cut, K-means and LRR together. And by solving an integrated optimization problem, SCSLRR can get the best subspace segmentation results. We use four image databases and Hopkins 155 database to evaluate the performances of SCSLRR and some related algorithms. The experimental results show that SCSLRR is a stable and fast convergent algorithm which also dominates the related algorithms.

\section{Acknowledgement}

The authors thank anonymous reviewers for their constructive comments on this paper. This work is supported by the National Science Foundation of China (Nos. 61203240, 61202022), the National Science Foundation of Shanghai (No. 13ZR1455600) and the Innovation Program of Shanghai Municipal Education Commission (14YZ102).

\section{Appendix A}

The detailed updating approach for $\mathbf{J}, \mathbf{W}, \mathbf{Z}, \mathbf{E}$ in Eq.(11) are stated as follows:

1. Update $\mathbf{J}$ with other fixed variables. We drop the irrelevant terms w.r.t. J in Eq.(10), then in the $k$-th iteration of Algorithm 1, we have

$$
\begin{aligned}
\mathbf{J}_{k+1} & =\arg \min _{\mathbf{J}_{k}}\left\|\mathbf{J}_{k}\right\|_{*}+<\mathbf{Y}_{2}^{k}, \mathbf{Z}_{k}-\mathbf{J}_{k}>+\mu_{k} / 2\left\|\mathbf{Z}_{k}-\mathbf{J}_{k}\right\|_{F}^{2} \\
& =\arg \min _{\mathbf{J}_{k}}\left\|\mathbf{J}_{k}\right\|_{*}+1 / 2\left\|\mathbf{J}_{k}-\left(\mathbf{Z}_{k}+\mathbf{Y}_{2}^{k} / \mu_{k}\right)\right\|_{F}^{2}
\end{aligned}
$$

then $\mathbf{J}_{k+1}=\mathbf{U}_{k} \boldsymbol{\Theta}_{1 / \mu_{k}}\left(\mathbf{S}_{k}\right) \mathbf{V}_{k}^{T}$, where $\mathbf{U}_{k} \mathbf{S}_{k} \mathbf{V}_{k}^{T}$ is the SVD of matrix $\mathbf{Z}_{k}+$ $\mathbf{Y}_{2}^{k} / \mu_{k}, \boldsymbol{\Theta}$ is the singular value thresholding operator[44]. 
2. Update $\mathbf{W}$ with other fixed variables. Collect the relevant terms w.r.t. $\mathbf{W}$ in Eq.(10), then in the $k$-th iteration of Algorithm 1, we have:

$$
\begin{aligned}
\mathbf{W}_{k+1} & =\hat{\lambda_{1}} \arg \min _{\mathbf{W}_{k}}\left\|\mathbf{M} \odot \mathbf{W}_{k}\right\|_{1}+<\mathbf{Y}_{3}^{k}, \mathbf{Z}_{k}-\mathbf{W}_{k}>+\mu_{k} / 2\left\|\mathbf{Z}_{k}-\mathbf{W}_{k}\right\|_{F}^{2}, \\
& =\hat{\lambda_{1}} / \mu_{k} \arg \min _{\mathbf{W}_{k}}\left\|\mathbf{M} \odot \mathbf{W}_{k}\right\|_{1}+1 / 2\left\|\mathbf{W}_{k}-\left(\mathbf{Z}_{k}+\mathbf{Y}_{3}^{k} / \mu_{k}\right)\right\|_{F}^{2},
\end{aligned}
$$

then $\left[\mathbf{W}_{k+1}\right]_{i j}=\Psi_{\varepsilon_{i j}}\left(\left[\mathbf{Z}_{k}+\mathbf{Y}_{3}^{k} / \mu_{k}\right]_{i j}\right)$, where $\Psi_{\varepsilon}(x)=\max (x-\varepsilon, 0)+\min (x+$ $\varepsilon, 0)$ and $\varepsilon_{i j}=\hat{\lambda_{1}} / \mu_{k}[\mathbf{M}]_{i j}$.

3. Update $\mathbf{Z}$ with other fixed variables. Similar to the previous processing, in the $k$-th iteration of Algorithm 1, we have:

$$
\begin{aligned}
\mathbf{Z}_{k+1}= & \arg \min _{\mathbf{Z}_{k}}\left\|\mathbf{X}-\mathbf{X} \mathbf{Z}_{k}-\mathbf{E}_{k}+\mathbf{Y}_{1}^{k} / \mu_{k}\right\|_{F}^{2}+\left\|\mathbf{J}_{k+1}-\left(\mathbf{Z}_{k}+\mathbf{Y}_{2}^{k} / \mu_{k}\right)\right\|_{F}^{2} \\
& +\left\|\mathbf{W}_{k+1}-\left(\mathbf{Z}_{k}+\mathbf{Y}_{3}^{k} / \mu_{k}\right)\right\|_{F}^{2}
\end{aligned}
$$

then $\mathbf{Z}_{k+1}=\left(\mathbf{X}^{T} \mathbf{X}+2 \mathbf{I}_{n}\right)^{-1}\left(\mathbf{X}^{T}\left(\mathbf{X}-\mathbf{E}_{k}+\mathbf{Y}_{1}^{k} / \mu_{k}\right)+\mathbf{J}_{k+1}-\mathbf{Y}_{2}^{k} / \mu_{k}+\mathbf{W}_{k+1}-\right.$ $\left.\left.\mathbf{Y}_{3}^{k} / \mu_{k}\right)\right)$.

4. Update $\mathbf{E}$ with other fixed variables. Negelect the irrelevant terms w.r.t. E in Eq.(10), then in the $k$-th iteration of Algorithm 1, we have:

$$
\begin{aligned}
\mathbf{E}_{k+1} & =\arg \min _{\mathbf{E}_{k}} \lambda_{2}\left\|\mathbf{E}_{k}\right\|_{2,1}+<\mathbf{Y}_{1}^{k}, \mathbf{X}-\mathbf{X} \mathbf{Z}_{k+1}-\mathbf{E}_{k}>+\left\|\mathbf{X}-\mathbf{X} \mathbf{Z}_{k+1}-\mathbf{E}_{k}\right\|_{F}^{2} \\
& =\arg \min _{\mathbf{E}_{k}} \lambda_{2} / \mu_{k}\left\|\mathbf{E}_{k}\right\|_{2,1}+\left\|\mathbf{E}_{k}-\left(\mathbf{X}-\mathbf{X} \mathbf{Z}_{k+1}+\mathbf{Y}_{1}^{k} / \mu_{k}\right)\right\|_{F}^{2}
\end{aligned}
$$

The above problem could be solved by following the Lemma presented in [3].

Lemma 1. Let $\mathbf{Q}=\left[\mathbf{q}_{1}, \mathbf{q}_{2}, \cdots, \mathbf{q}_{i}, \cdots\right]$ be a given matrix. If the optimal solution to

$$
\min _{\mathbf{T}} \nu\|\mathbf{T}\|_{2,1}+\frac{1}{2}\|\mathbf{T}-\mathbf{Q}\|_{F}^{2}
$$

is $\mathbf{T}^{*}$, then the $i$-th column of $\mathbf{T}^{*}$ is

$$
\mathbf{T}^{*}(:, i)= \begin{cases}\frac{\left\|\mathbf{q}_{i}\right\|_{2}-\nu}{\left\|\mathbf{q}_{i}\right\|_{2}} \mathbf{q}_{i}, & \text { if } \nu<\left\|\mathbf{q}_{i}\right\|_{2} ; \\ 0, & \text { oterwise. }\end{cases}
$$




\section{References}

[1] S. Rao, R. Tron, R. Vidal, Y. Ma, Motion Segmentation in the Presence of Outlying, Incomplete, or Corrupted Trajectories. IEEE Transactions on Pattern Analysis and Machine Intelligence, 32(10) (2010) 1832-1845.

[2] Y. Ma, H. Derksen, W. Hong, J. Wright, Segmentation of Multivariate Mixed Data via Lossy Data Coding and Compression, IEEE transactions on pattern analysis and machine intelligence, 29 (2007) 1-17.

[3] G. Liu, Z. Lin, S. Yan, J. Sun, Y. Yu, Y. Ma, Robust Recovery of Subspace Structures by Low-Rank Representation, IEEE Transaction on Pattern Analysis and Machine Intelligence, 35 (2013) 171-184.

[4] R. Vidal, Subspace Clustering, IEEE Signal Process Magazine, 28(2) (2011) 52-68.

[5] T. Zhang, A. Szlam, Y. Wang, G. Lerman. Hybrid linear modeling via local best-fit flats. International Journal of Computer Vision, 100(3) (2012) 217-240.

[6] P. S. Bradley, O. L. Mangasarian, K-plane clustering, Journal of Global Optimization, 16(1) (2000) 23-32.

[7] K. Huang, Y. Ma, R. Vidal Minimum effective dimension for mixtures of subspaces: A robust GPCA algorithm and its applications. In IEEE Conference on Computer Vision and Pattern Recognition (CVPR), (2004) 631-638.

[8] Y. Ma, A. Y. Yang, H. Derksen, R. Fossum, Estimation of subspace arrangements with applications in modeling and segmenting mixed data. SIAM Review, 50(3) (2008) 413-458. 
[9] A. Leonardis, H. Bischof, J. Maver, Multiple eigenspaces. Pattern Recognition, 35(11) (2002) 2613-2627.

[10] Y. Ma, H. Derksen, W. Hong, J. Wright, Segmentation of multivariate mixed data via lossy coding and compression. IEEE Transactions on Pattern Analysis and Machine Intelligence, 29(9) (2007) 1546-1562.

[11] E. Elhamifar, R. Vidal, Sparse Subspace Clustering, CVPR, (2009).

[12] V.M. Patel, H.V. Nguyen, R. Vidal, Latent Space Sparse Subspace Clustering, ICCV, (2013) 225-232.

[13] C. Lu, J. Tang, M. Lin, L. Lin, S. Yan, Z. Lin, Correntropy Induced L2 Graph for Robust Subspace Clustering, ICCV, (2013) 1801-1808.

[14] G. Liu, Z. Lin, Y. Yu, Robust Subspace Segmentation by Low-Rank Representation, ICML, (2010).

[15] R. Vidal, P. Favaro, Low rank subspace clustering, Pattern Recognition Letters, 43 (2014) 47-61.

[16] L. Wei, A. Wu, J. Yin, Latent space robust subspace segmentation based on low-rank and locality constraints, Expert Systems with Applications, 42 (2015) 6598-6608.

[17] J. Shi, J. Malik, Normalized cuts and image segmentation, IEEE transactions on pattern analysis and machine intelligence, 22 (2000) 888-905.

[18] R. O. Duda, P.E. Hart, D.G. Stork, Pattern classification (second ed.), Wiley-Interscience (2000). 
[19] L. Qiao, S. Chen, X. Tan, Sparsity Preserving Discriminant Analysis for Single Training Image Face Recognition, Pattern Recognition Letters, 31 (2010) 422-429.

[20] J. Wright, A.Y. Yang, A. Ganesh, S.S. Sastry, Y. Ma, Robust Face Recognition via Sparse Representation, IEEE transactions on pattern analysis and machine intelligence, 31 (2009) 210-227.

[21] E. Elhamifar, R. Vidal, Sparse Subspace Clustering: Algorithm, Theory, and Applications. IEEE transactions on pattern analysis and machine intelligence, 35(11) (2013) 2765-2781.

[22] L. Zhuang, H. Gao, Z. Lin, Y. Ma, X. Zhang, N. Yu, Non-negative low rank and sparse graph for semi-supervised learning, CVPR, (2012) 2328-2335.

[23] M. Zhao, L. Jiao, J. Feng, T. Liu, A simplified low rank and sparse graph for semi-supervised learning, Neurocomputing, 140 (2014) 84-96.

[24] S. Li, Y. Fu, Low-Rank Coding with b-Matching Constraint for SemiSupervised Classification, IJCAI, (2013).

[25] K. Tang, R. Liu, J. Zhang, Structure-Constrained Low-Rank Representation, IEEE TRANSACTIONS ON NEURAL NETWORKS AND LEARNING SYSTEMS, 25 (2014) 2167-2179.

[26] X. Lu, Y. Wang, and Y. Yuan, Graph-Regularized Low-Rank Representation for Destriping of Hyperspectral Images. IEEE Transaction on Geoscience and Remote Sensing, 2013. 51(7-1): p. 4009-4018.

[27] G. Liu, S. Yan, Latent Low-Rank Representation for Subspace Segmentation and Feature Extraction, ICCV, (2011). 
[28] M. Yin, J. Gao, Z. Lin, Q. Shi and Y. Guo, Dual Graph Regularized Latent Low-Rank Representation for Subspace Clustering. IEEE Trans Image Process, 2015. 24(12): p. 4918-33.

[29] R. Liu, Z. Lin, F.D.l. Torre, Z. Su, Fixed-Rank Representation for Unsupervised Visual Learning, CVPR, (2012).

[30] M. Yang, L. Zhang, J. Yang, D. Zhang, Robust Sparse Coding for Face Recognition, CVPR, (2011).

[31] J. Chen, J. Yang, Robust Subspace Segmentation Via Low-Rank Representation, IEEE TRANSACTIONS ON CYBERNETICS, 44 (2014) 1432-1445.

[32] J. Wright, Y. Peng, Y. Ma, A. Ganesh, S. Rao, Robust Principal Component Analysis: Exact Recovery of Corrupted Low-Rank Matrices by Convex Optimization, NIPS, (2009).

[33] J. Jiang, J. Yang, Y. Cui, L. Luo, Mixed noise removal by weighted low rank model, Neurocomputing, 151 (2015) 817-826.

[34] C.Y. Lu, H. Min, Z.Q. Zhao, L. Zhu, D.S. Huang, S. Yan, Robust and Efficient Subspace Segmentation via Least Squares Regression, ECCV, (2012).

[35] H. Hu, Z. Lin, J. Feng, J. Zhou, Smooth Representation Clustering, CVPR, (2014).

[36] J.F. Cai, E. J. Candes, Z. Shen, A singular value thresholding algorithm for matrix completion. SIAM Journal on Optimization, (4) (2010) 19561982. 
[37] C. Ding, X. He, K-means clustering via principal component analysis. ICML, 2004.

[38] F. Samaria, A. Harter, Parameterisation of a stochastic model for human face identification, In: Proceedings of second IEEE workshop applications of computer vision, 1994.

[39] K.C. Lee, J. Ho, D. Driegman, Acquiring linear subspaces for face recognition under variable lighting. IEEE Transactions on Pattern Analysis and Machine Intelligence, 27(5) (2005) 684C698.

[40] R. Tron, R. Vidal, A benchmark for the comparison of 3-D motion segmentation algorithms. IEEE International Conference on Computer Vision and Pattern Recognition (ICCV), (8) (2007).

[41] A.M. Martinez, R. Benavente, The AR face database, CVC, Univ. Autonoma Barcelona, Barcelona, Spain, Technical Report 24, (1998).

[42] P.N. Belhumeur, J.P. Hespanha, D.J. Kriegman, Eigenfaces vs. Fisherfaces: Recognition using class specific linear projection. IEEE Trans Pattern Anal Mach Intell, 1997. 19(7): p. 711-720.

[43] M. Yin, J. Gao, and Z. Lin, Laplacian Regularized Low-Rank Representation and Its Applications. IEEE Transactions on Pattern Analysis and Machine Intelligence, 2015: p. 1-1.

[44] J. F. Cai, E. J. Candes, Z. Shen, A singular value thresholding algorithm for matrix completion. SIAM Journal on Optimization, 2010, 20(4):1956C1982. 
[45] C. Yan, Y. Zhang, F. Dai, X. Wang, L. Li, Q. Dai, Parallel deblocking filter for HEVC on many-core processor, Electronics Letters, 2014 50(5):367-368.

[46] C. Yan, Y. Zhang, J. Xu, F. Dai, L. Li, Q. Dai, F. Wu, A Highly Parallel Framework for HEVC Coding Unit Partitioning Tree Decision on Manycore Processors. IEEE Signal Process. Lett., (2014) 21(5): 573-576.

[47] C. Yan, Y. Zhang, J. Xu, F. Dai, J. Zhang, Q. Dai, F. Wu, Efficient Parallel Framework for HEVC Motion Estimation on Many-Core Processors. IEEE Trans. Circuits Syst. Video Techn., 2014: 24(12): 20772089.

[48] J. Duchi, Y. Singer, Boosting with structural sparsity, in Proc. ICML, 2009, pp. 297C304.

[49] L. Li, S. Jiang, Q. Huang, Learning Hierarchical Semantic Description Via Mixed-Norm Regularization for Image Understanding. IEEE Transactions on Multimedia, 2012 14(5): 1401-1413.

[50] C.-G. Li and R. Vidal, Structured Sparse Subspace Clustering: A Unified Optimization Framework. CVPR, 2015 